\title{
Q.
QNEEN'S
UNIVERSITY
BELFAST
}

\section{A Dynamic Highly Reliable SRAM-Based PUF Retaining Memory Function}

Zhang, H., Wang, C., Yan, C., Cui, Y., Gu, C., O'Neill, M., \& Liu, W. (2021). A Dynamic Highly Reliable SRAMBased PUF Retaining Memory Function. In 2021 IEEE International Symposium on Circuits and Systems (ISCAS) ( IEEE International Symposium on Circuits and Systems (ISCAS): Proceedings). Institute of Electrical and Electronics Engineers Inc..

Published in:

2021 IEEE International Symposium on Circuits and Systems (ISCAS)

Document Version:

Peer reviewed version

Queen's University Belfast - Research Portal:

Link to publication record in Queen's University Belfast Research Portal

Publisher rights

(c) 2021 IEEE.

This work is made available online in accordance with the publisher's policies. Please refer to any applicable terms of use of the publisher.

\section{General rights}

Copyright for the publications made accessible via the Queen's University Belfast Research Portal is retained by the author(s) and / or other copyright owners and it is a condition of accessing these publications that users recognise and abide by the legal requirements associated with these rights.

Take down policy

The Research Portal is Queen's institutional repository that provides access to Queen's research output. Every effort has been made to ensure that content in the Research Portal does not infringe any person's rights, or applicable UK laws. If you discover content in the Research Portal that you believe breaches copyright or violates any law, please contact openaccess@qub.ac.uk. 


\section{A Highly Reliable SRAM-Based PUF Retaining Memory Function}

\begin{abstract}
In this paper, a novel highly reliable SRAM based Physical Unclonable Function (PUF) is proposed, which retains the memory function. The mismatch of NMOS is extracted during discharge process and amplified by the cross-coupled inverter to generate a response. At the beginning of discharge process, the NMOSs are biased at sub-threshold region, which can improve the reliability and stability. The proposed PUF is designed in a 40nm CMOS process and each bit cell only consumes $4.98 \mu \mathrm{m}^{2}$ $\left(3112 F^{2}\right)$. Post simulation shows that the bit error rate (BER) deterioration is $0.96 \%$ per $0.1 \mathrm{~V}, 0.36 \%$ per $10^{\circ} \mathrm{C}$ with temperature changes from $-40^{\circ} \mathrm{C}$ to $80^{\circ} \mathrm{C}$ temperature and supply voltage changes from $0.9 \mathrm{~V}$ to $1.3 \mathrm{~V}$. It achieves $1.8 \%$ native instability through the simulation. Meanwhile, the proposed PUF can retains memory function after response generated.

Index Terms-Physical unclonable function (PUF), SRAM, high reliability, subthreshold.
\end{abstract}

\section{INTRODUCTION}

With the rapid development of the Internet of Things (IoTs), hardware security has become a critical issue. Traditional hardware encryption technology stores the key in NonVolatile Memory (NVM), which is vulnerable to attack such as side channel and reverse engineering [1]. As a hardware security primitive, physical unclonable function (PUF) can extract the random process variations during manufacturing process as a unique identification, which likes a fingerprint. Due to the manufacturing variations, it is to be measured and the PUF identification would disappear when it powers down. Therefore, PUF can be used as a emerging solution to protect important information from hackers [2].

However, the responses of PUF are sensitive to environmental noise, temperature variation and supply voltage, which limits PUF using as a key generator. Besides, with the increase of PUF using time, the aging effect leads to output data overturning. As a result, the bit error rate (BER) increases [3]. High BER needs complicated error correction module to correct the error, which consumes much hardware resources [4]. This is not desired for a low-cost PUF design.

SRAM PUF can generate the responses without additional circuit. However, the traditional SRAM PUF shows nearly $30 \%$ instability [5]. In order to reduce the area consumption, a novel SRAM PUF with two bits per PUF cell is proposed in [6], but the native instability is still high, which is $17 \%$. In [7], EE SRAM PUF is proposed to magnify the mismatch, thus greatly reducing the BER. However, the power consumption of EE SRAM PUF is high because the transistor works at the saturation region, resulting in large static power. A compact PUF is presented in [8], which can improve the reliability against to temperature variation, but the voltage reliability is not improved.

In order to reduce the BER and power consumption of SRAM PUF, this paper utilizes the difference of subthreshold discharge current to extract circuit mismatch. Specifically, the main improvements of this work are shown as follows:

- A new PUF based on SRAM with high stability and reliability is proposed. The stability is improved by starting up with an elaborately designed enabling signal instead of the supply voltage.

- The power consumption of the proposed SRAM PUF is decreased by controling the enabling signals sequence, which can reduce the current effectively.

- The memory function is retained by configuring the enabling signals to a specific state.

The rest of this paper is organized as follows: Section II analyzes characteristic of subthreshold discharge current; Section III presents the proposed SRAM PUF and the layout of overall PUF architecture. The results and comparison of proposed PUF is presented in Section IV. The conclusion is given in Section $\mathrm{V}$.

\section{BASIS OF SUB-THRESHOLD CURRENT}

When the MOS transistor works in the saturation and sub threshold region, the current are expressed as follows:

$$
\begin{gathered}
I_{s a t}=\mu C_{o x}\left(\frac{W}{L}\right)\left(V_{g s}-V_{t h}\right)^{2} \\
I_{s u b}=\mu C_{o x}\left(\frac{W}{L}\right) \exp \left(\frac{V_{g s}-V_{t h}}{V_{t}}\right)\left(1-\exp \left(-\frac{V_{d s}}{V_{t}}\right)\right)
\end{gathered}
$$

where $\mu$ is the carrier mobility of NMOS, $C_{o x}$ is the sheet oxide capacitance density, $W / L$ is the width to length ratio of NMOS transistor, $V_{t h}$ is the threshold voltage, and $V_{t}$ is the thermal voltage. $I_{\text {sat }}$ represents the current in the saturation region, while $I_{s u b}$ means the current at the subthreshold. Since $V_{d s}$ starts discharging from $V_{d d}, V_{d s}$ is much greater than $V_{t}$, thus $\exp \left(-V_{d s} / V_{t}\right)$ be ignored.

Fig. 1 shows the circuit schematic of current mirror. The gate voltage $V_{g s}$ controls the current passing through the transistor. When the switch $\mathrm{S}$ turns on, $V_{d s}$ would be charged to $V_{d d}$. When the switch $\mathrm{S}$ turns off, NMOS starts to discharge and the $V_{d s}$ decreases continuously. According to the discharge principle of RC circuit and the mismatch between M1 and M2, the voltage of $V_{d s 1}$ and $V_{d s 2}$ would be different over discharge time, as given by 


$$
\frac{V_{d s 1}}{V_{d s 2}}=\exp \left(-\frac{t}{C}\left(\frac{I_{1}}{V_{d s 1}}-\frac{I_{2}}{V_{d s 2}}\right)\right)
$$

where $\mathrm{C}$ is the capacitance of the circuit load connected to the drain of M1 or M2. Since the value is slightly affected by the mismatch between M1 and M2, it can be assumed that the load capacitance values are equal. $I_{1}$ and $I_{2}$ represent the discharge current over M1 and M2, respectively. When $V_{g s}$ is different, the transistor will work in the saturation region or sub-threshold region. The discharge current will also change.

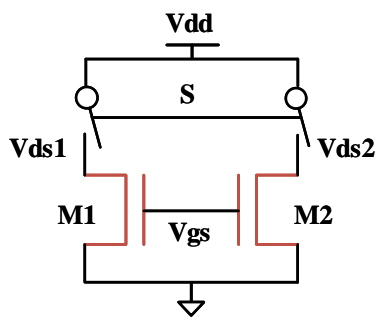

Fig. 1. Schematic of current mirror.

It can be noted in (1) that the sub-threshold current is exponentially related to the threshold voltage. Thus its value is more sensitive to the change of the threshold voltage compared with saturation value in (2). From (3), it can be seen that the value of $V_{d s 1} / V_{d s 2}$ in sub-threshold region has more significant difference than it in saturation region with the same mismatch. Therefore, the stability of PUF circuit can be improved. Furthermore, the discharge current in subthreshold is very small, resulting in low power consumption.

Meanwhile, (3) shows that the ratio of $V_{d s 1} / V_{d s 2}$ is not affected by the supply voltage, which can improve voltage reliability.

\section{THE PRoposed PUF}

\section{A. PUF Cell Based on SRAM}

The schematic of the proposed PUF cell is shown in Fig. 2. The circuit consists of a traditional SRAM cell and several switch transistors. The proposed PUF cell is controlled by EN and ENB. In the initial state, EN set to 0 and ENB set to 1. The gate voltages of M3 and M4 are pulled up to $V_{d d}$, and the gate voltages of M1 and M2 are pulled down to the ground. In this case, M7 and M8 are turned off to eliminate the static current. Then, the voltages $V_{a}$ and $V_{b}$ are discharged from $V_{d d}$ through M1 and M2 while EN is set to 1 and ENB changes inversely. The values of $V_{g s 1}$ and $V_{g s 2}$ are increased from 0 in the process. Because of the voltage stabilizing effect of M7 and M8, $V_{g s 1}$ and $V_{g s 2}$ are always smaller than the threshold voltage, which keeps the whole discharge process in the subthreshold region. The mismatch between M1 and M2 leads to the difference of $V_{a}$ and $V_{b}$, and it can be amplified by the cross coupling inverters. It is worth nothing that the proposed PUF cell can work as a traditional SRAM when EN remains 1 and ENB is 0.

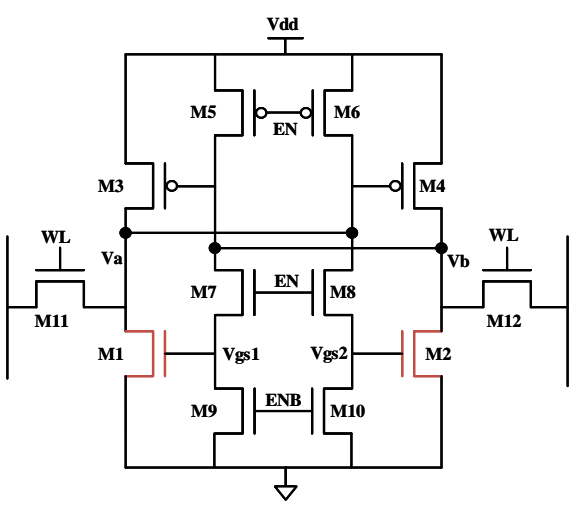

Fig. 2. Schematic of the proposed PUF cell.

The transient simulation waveforms of proposed PUF cell are shown in the Fig. 3. It can be found from the figure that when EN changes from 0 to 1 , the voltage values of $V_{g s 1}$ and $V_{g s 2}$ increase gradually. However, due to the existence of M7 and M8, the voltage remains coincident. At the same time, the voltages of $V_{a}$ and $V_{b}$ decrease gradually due to the discharge. Furthermore, the voltage values of $V_{g s 1}$ and $V_{g s 2}$ are smaller than the threshold voltage, which keeps M1 and M2 working in the subthreshold region. The difference between $V_{a}$ and $V_{b}$ in the discharge process expands gradually. M1, M2, M3 and M4 amplify $V_{a}$ and $V_{b}$ to 0 or 1 , respectively. Then the response can be read out through the word line, which controlled by WL.

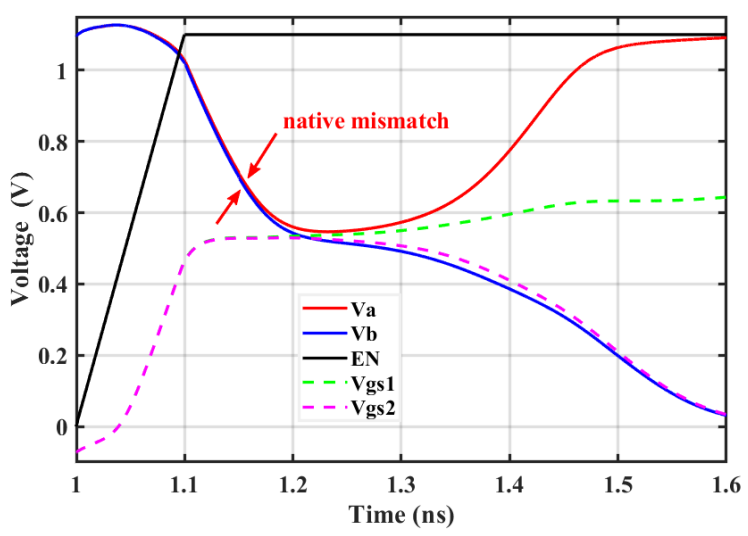

Fig. 3. Transient simulation waveforms of PUF cell.

\section{B. Time Strategy}

As shown in Fig. 2, the following situation will occur if ENB is enabled after EN. The conduction state of M9 and M10 before ENB is enabled causes the voltages of $V_{a}$ and $V_{b}$ to drop and makes M3 and M4 in the saturation region, which generates a DC path from the supply voltage to the ground. After ENB is enabled, M9 and M10 turns off, and $V_{a}$ and $V_{b}$ are charged gradually through M3 and M4. However, the 
high energy consumption situation is undesired and is not the sub-threshold discharge state we need.

In order to reduce the power consumption and stabilize the output, a time strategy schematic of controling enable signal is proposed. As shown in the Fig. 4. The schematic consists of an array of inverter pairs. Each pair of inverters can ensure the correct sequence of signals and control $2 \times 4$ PUF cells. A total of $8 \times 8$ PUF cells can be controlled.

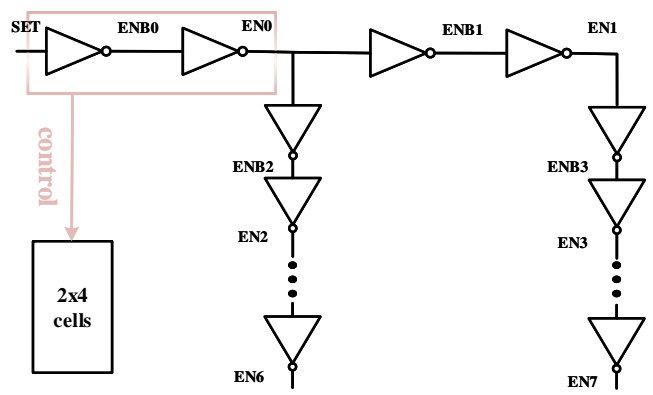

Fig. 4. Schematic of the enable module controling $8 \times 8$ cells.

\section{Overall Architecture and Layout}

The proposed PUF employs a array architecture, as shown in the Fig. 5(a), which is similar as SRAM. The decoder can choose the position of the PUF cell through the controling word line. In order to reduce the area consumption, hierarchical decoding is adopted. When reading out the PUF enabled response, the circuit should be precharged, which is controlled by the signal PC. At the end of the precharge, the word line WL selects the PUF cell output, which is amplified by the amplifier. The enable module provides correct timing control for the whole circuit.

The proposed implementation was designed using a standard $40 \mathrm{~nm}$ CMOS process. Fig. 5(b) shows both the layout of enable module and PUF core, which features a compact overall silicon area of $1,540 \mu \mathrm{m}^{2}$. Each PUF cell occupies an area of $4.98 \mu \mathrm{m}^{2}$. The peripheral circuitries such as the decoders, SA, and the per-charge circuit were accomplished by schematic, which did not affected the results of responses.

\section{Simulation Results And Comparison}

In order to better evaluate the performance of the proposed PUF, the PUF layout Monte Carlo simulation results is exhibited. The uniqueness, stability and reliability of the proposed PUF are analyzed by post-simulation. Finally, the advantages of the proposed PUF are discussed.

\section{A. Uniqueness}

Uniqueness can be expressed by hamming distance between chips. The inter-chip Hamming distance (Inter-HD) is expressed as the HD of responses from different PUF chips in the same environment. In order to evaluate the uniqueness of PUF, we simulated 50 times of Monte Carlo simulation on the

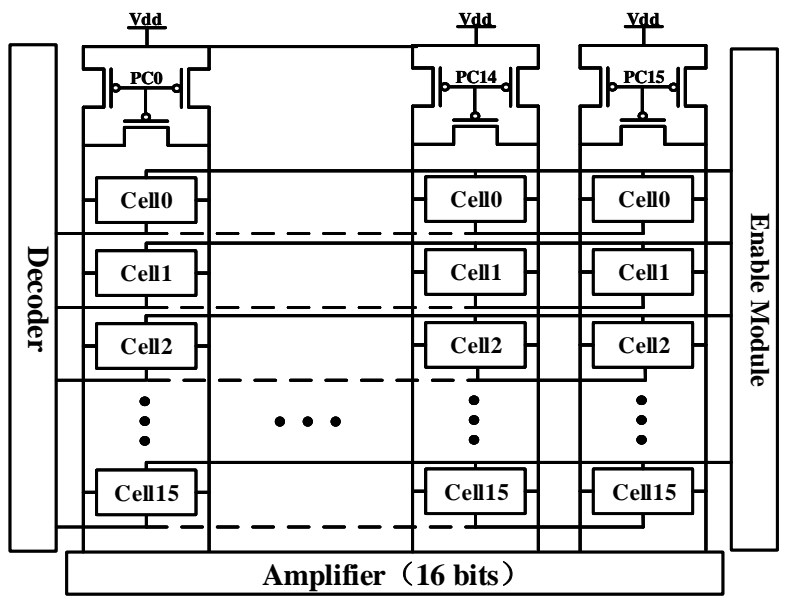

(a)

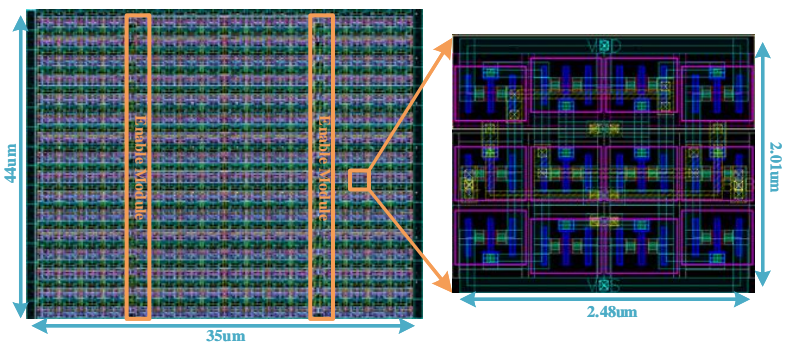

(b)

Fig. 5. (a) Overall archiecture of the proposed PUF; (b) Layout of the proposed PUF core.

proposed PUF layout at $27^{\circ} \mathrm{C}$ and $1.1 \mathrm{~V}$. The result is shown in Fig. 6 that Inter-HD exhibits a Gaussian distribution with $\mu=0.49$ and $\sigma=0.0676$.

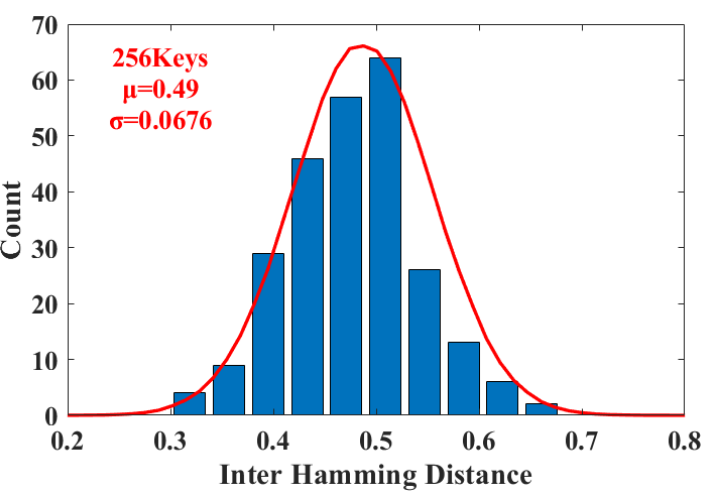

Fig. 6. Layout simulation of inter-HDs across 256 keys. 


\section{B. Stability}

Due to the influence of environmental factors such as noise, PUF responses cannot remain constant under multiple measurements even if the same chip. Stability can be expressed by BER. In order to better simulate the actual PUF chip environment, we performed 2,000 noise simulations at a noise frequency between $100 \mathrm{KHz}$ to $500 \mathrm{MHz}$. The results show that the native BER of the pre-simulation is $0.8 \%$, and the post-simulation is $1.8 \%$. After traditional temporal majority voting (TMV) error correction, the post-simitation instability is reduced to $0.3 \%$.

\section{Reliability}

The proposed PUF's reliability is affected by environmental temperature and supply voltage variations. We simulated the temperature reliability of $-40^{\circ} \mathrm{C} \sim 80^{\circ} \mathrm{C}$ and the voltage reliability of $0.9 \mathrm{~V} \sim 1.3 \mathrm{~V}$. Furthermore, we compared with the results of native BER in [7] and [8], as shown in the Fig. 7. The results show that the proposed PUF has obvious advantages in voltage and temperature reliability. For per $0.1 \mathrm{~V}$ voltage change, the average BER deteriorates by $0.98 \%$, and the temperature changes per $10^{\circ} \mathrm{C}$, the BER increases by $0.36 \%$.

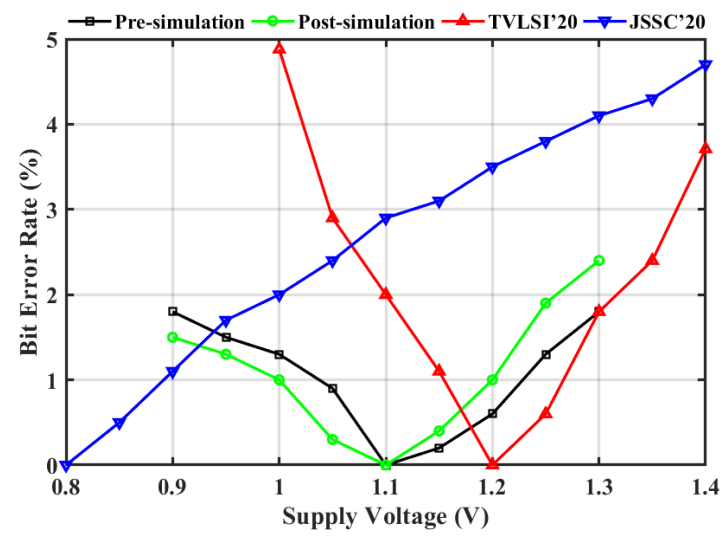

(a)

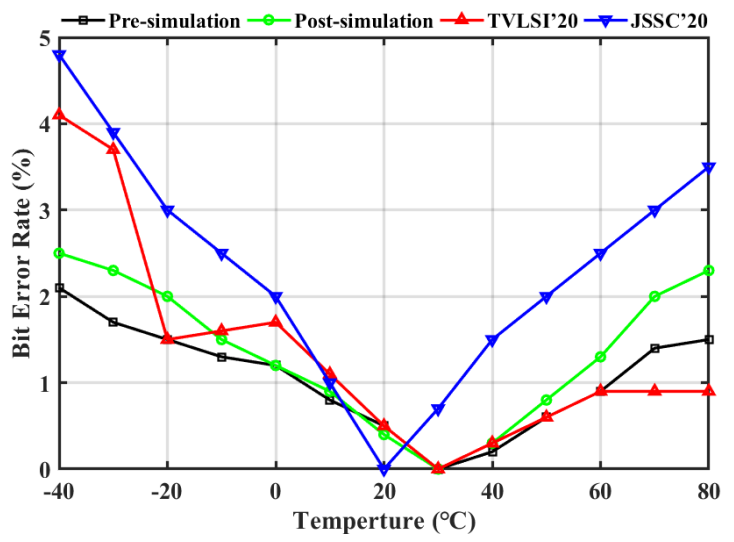

(b)

Fig. 7. (a) Voltage relibility between $0.8 \mathrm{~V}$ to $1.3 \mathrm{~V}$; (b) Temperature relibility between $-40^{\circ} \mathrm{C}$ to $80^{\circ} \mathrm{C}$.
TABLE I

COMPARISON WITH THE STATE-OF-THE-ART WEAK PUF

\begin{tabular}{|c|c|c|c|c|c|}
\hline & This Work & {$[7]$} & {$[8]$} & {$[9]$} & {$[10]$} \\
\hline Technology & $40 \mathrm{~nm}$ & $130 \mathrm{~nm}$ & $65 \mathrm{~nm}$ & $22 \mathrm{~nm}$ & $14 \mathrm{~nm}$ \\
\hline Structure & SRAM & $\begin{array}{c}\text { EE } \\
\text { SRAM }\end{array}$ & $\begin{array}{c}\text { Cross- } \\
\text { coupled }\end{array}$ & SRAM & SRAM \\
\hline bitcell size $\left(F^{2}\right)$ & 3112 & 373 & 1036 & 9628 & 9387 \\
\hline Unstable Bit $(\%)$ & $\mathbf{1 . 8}$ & 2.14 & 2.64 & 30 & 26.38 \\
\hline Temp. Range( $\left({ }^{\circ} \mathrm{C}\right)$ & $-40 \sim 80$ & $-40 \sim 120$ & $-50 \sim 150$ & N/A & N/A \\
\hline Volt. Range $(\mathrm{V})$ & $0.9 \sim 1.3$ & $0.8 \sim 1.4$ & $1.0 \sim 1.4$ & N/A & N/A \\
\hline BER per $10^{\circ} \mathrm{C}(\%)$ & $\mathbf{0 . 3 6}$ & 0.75 & 0.26 & N/A & N/A \\
\hline BER per 0.1V(\%) & $\mathbf{0 . 9 8}$ & 0.78 & 2.34 & N/A & N/A \\
\hline Uniqueness & 49 & $\sim 0.5$ & 49.53 & 50.001 & 48.6 \\
\hline Core Energy(fJ/bit) & $\mathbf{1 3 . 2 5}$ & 128 & 2980 & 13 & 4 \\
\hline $\begin{array}{c}\text { Memory } \\
\text { Function }\end{array}$ & Yes & No & No & \multirow{2}{*}{ No } & No \\
\hline
\end{tabular}

\section{Comparison}

Table I provides a comprehensive comparison of our proposed PUF against the reported previous works. It shows that the stability of the proposed PUF is the best compared to [7], [8], [9], [10]. The temperature reliability of the proposed PUF is not much different from the optimal value of [8]. Meanwhile, the voltage reliability is improved by more than $50 \%$. Compared with [7], the temperature reliability has obvious advantages, while the voltage reliability has not been reduced much. It is particularly pointed out that the power consumption of [7] is measured at $0.8 \mathrm{~V}$ supply voltage, and other comparative works is measured at the standard voltage of the corresponding process. On the other hand, considering the characteristics of less energy consumption in the more advanced process, the energy consumption of our proposed PUF is the smallest, which is a very competitive circuit. One of the most important contributions of this work is that our improved SRAM-based PUF still has memory function, so our PUF can not only be used as a key generator, but also can be used with traditional SRAM as a cache. Furthermore, the proposed PUF area is compact comparing with [9], [10].

\section{CONCLUSION}

In this paper, a SRAM-based PUF cell is proposed, which utilizes subthreshold discharge to improve voltage reliability and temperature reliability. In addition, a new time strategy is designed to reduce PUF power consumption. A supersymmetrical PUF cell layout is designed and the postsimulation performance is not reduced. Meanwhile, the proposed PUF retains the memory function, which has a wide range of application scenarios.

\section{REFERENCES}

[1] Yijun Cui, Qingqing Ma, Chongyan Gu, Yue Fang, Chenghua Wang, Maire O'Neill and Weiqiang Liu, "Lightweight Modeling Attacks Resistant Multiplexer Based Multi-PUF (MM-PUF) Design on FPGA," Electronics, vol. 9, no. 5,815, 2020.

[2] Chongyan Gu, Weiqiang Liu, Yijun Cui, Neil Hanley, Maire O’Neill, and Fabrizio Lombardi, “A Flip-Flop Based Arbiter Physical Unclonable Function (APUF) Design with High Entropy and Uniqueness for FPGA Implementation," IEEE Transactions on Emerging Topics in Computing, pp. 1-1, 2019. 
[3] R. Maes and V. van der Leest, "Countering the Effects of Silicon Aging on SRAM PUFs," in Proc. IEEE International Symposium on HardwareOriented Security and Trust (HOST), pp. 148-153, 2014.

[4] W. Liu, L. Zhang, Z. Zhang, C. Gu, C. Wang, M. O'neill, and F. Lombardi, "XOR-Based Low-Cost Reconfigurable PUFs for IoT Security," ACM Trans. Embed. Comput. Syst., vol. 18, no. 3, 2019.

[5] S. Chellappa and L. T. Clark, "SRAM-Based Unique Chip Identifier Techniques," IEEE Transactions on Very Large Scale Integration (VLSI) Systems, vol. 24, no. 4, pp. 1213-1222, 2016.

[6] Y. Shifman, A. Miller, Y. Weizman, A. Fish, and J. Shor, "An SRAM PUF with 2 Independent Bits/Cell in 65nm," in Proc. IEEE International Symposium on Circuits and Systems (ISCAS), pp. 1-5, 2019.

[7] K. Liu, Y. Min, X. Yang, H. Sun, and H. Shinohara, "A 373-F" 0.21\%-Native-BER EE SRAM Physically Unclonable Function With 2D Power-Gated Bit Cells and $V_{\text {SS }}$ Bias-Based Dark-Bit Detection," IEEE Journal of Solid-State Circuits, vol. 55, no. 6, pp. 1719-1732, 2020.

[8] Q. Zhao, Y. Wu, X. Zhao, Y. Cao, and C. Chang, "A 1036- $\mathrm{F}^{2} / \mathrm{Bit}$ High Reliability Temperature Compensated Cross-Coupled ComparatorBased PUF," IEEE Transactions on Very Large Scale Integration (VLSI) Systems, vol. 28, no. 6, pp. 1449-1460, 2020

[9] S. K. Mathew, S. K. Satpathy, M. A. Anders, H. Kaul, S. K. Hsu, A. Agarwal, G. K. Chen, R. J. Parker, R. K. Krishnamurthy, and V. De, "A $0.19 \mathrm{pJ} / \mathrm{b}$ PVT-Variation-Tolerant Hybrid Physically Unclonable Function Circuit for $100 \%$ Stable Secure Key Generation in 22nm CMOS," in Proc. IEEE International Solid-State Circuits Conference Digest of Technical Papers (ISSCC), pp. 278-279, 2014.

[10] S. Satpathy, S. K. Mathew, V. Suresh, M. A. Anders, H. Kaul, A. Agarwal, S. K. Hsu, G. Chen, R. K. Krishnamurthy, and V. K. De, "A 4-fJ/b Delay-Hardened Physically Unclonable Function Circuit With Selective Bit Destabilization in 14-nm Trigate CMOS," IEEE Journal of SolidState Circuits, vol. 52, no. 4, pp. 940-949, 2017. 